\title{
Rigid aspheric smoothing tool for mid- spatial frequency errors on aspheric or freeform optical surfaces
}

\author{
Guoyu Yu ${ }^{1 *}$ (D) Lunzhe $\mathrm{Wu}^{2}$, Xing Su${ }^{3}$, Yuancheng $\mathrm{Li}^{1}$, Ke Wang ${ }^{4}$, Hongyu $\mathrm{Li}^{1,5}$ and David Walker ${ }^{1,6,7}$
}

\begin{abstract}
In order to remove mid-spatial frequency errors on aspheric and freeform surfaces, we have developed an aspheric smoothing tool which, unusually, is rigid. This has been proved feasible in the special case where the abrasive grit size exceeds the aspheric misfit, providing a cushion. Firstly, experimental parameters were derived from simulation of Influence Functions regarding misfit between the tool and the surface, which leads to dynamic Influence functions. Then the experimental part was polished into an aspheric surface from generated spherical surface. Thirdly, the choice of tool's shape parameters was completed with optimisation of conic constant and tilt angle. The tool was machined into aspheric shape with a single-point cutter. Finally, experiments were carried out to compare this tool with a standard spherical smoothing tool. The results showed that this aspheric smoothing tool can removal mid-spatial errors effectively on aspheric surfaces.
\end{abstract}

Keywords: Mid-spatial frequency error, Freeform, Optical fabrication, Aspheric

\section{Introduction}

There has been considerable progress on advanced manufacturing of optical surfaces since the introduction of computer numerical controlled $(\mathrm{CNC})$ polishing machines. Compared with traditional manual intensive craftsmanship, these machines offered higher efficiency, repeatability and stability for optical manufacturing industry $[1,2]$. As a result, large aperture optical surfaces can be processed with very high form specifications and edge profiles [3-5]. This has enabled serial production of segmented mirrors used for very large telescopes $[6,7]$.

Despite the success in form figuring, there are issues with the $\mathrm{CNC}$ machines in dealing with the mid-spatial frequency (MSF) errors. This type of surface irregularity has been describes and studied extensively before [8-11] and research to control MSF errors reported e.g [12-15] The MSF errors originate from various sources. They may arise from a prior generating process where hardgrinding was used, or coarse loose-abrasive grits or wide

\footnotetext{
* Correspondence: g.yu@hud.ac.uk

${ }^{1}$ National Facility for Ultra Precision Surfaces, OpTIC Centre, University of Huddersfield, St. Asaph Business Park, Ffordd William Morgan, St Asaph, North Wales LL17 0JD, UK

Full list of author information is available at the end of the article
}

toolpath tracks were employed. Even with fine grinding, MSF errors occur when generating aspheric surfaces, where cup-wheel edge-contact is necessary. This is mainly due to mechanical vibration-modes, and exhibits varied spatial frequency features. Similar situations occurs in diamond-turning, where metal mirrors can be machined to high form accuracy. For large aperture metal mirrors, MSF error have been observed when aspheric surface are manufactured. This is mainly due to the requirement of servo movements of the cutting point for varying sag heights at each cycle of the toolpath. MSF errors can also occur at figuring processes, even though the input was an MSF-free surface. These MSF errors may be introduced by tool run-out, slurry starvation, or in metrology data-processing, such as errors in registration of the measurement with the part's physical surface. Such errors can limit convergence in figuring, and ultimately lead to product-failure should a super-smoothed surface be required for critical applications such as EUV photo-lithography. On the other hand, slope-errors associated with MSFs may compromise or prevent interferometric metrology of the surface to be processed. Such issues can be partially resolved by surface-smoothing, but there is clearly considerable 
scope to improve such techniques particularly when applied to aspheric or freeform surfaces.

Rigid smoothing tools are normally used for removing MSF errors on flat or spherical surfaces, and can then provide an input-quality suitable for reliable interferometry. For aspheres, semi-rigid tools have been extensively used, e.g. [16, 17], or various actively-deformed laps, e.g [18] The characteristics of non-Newtonian materials have also been extensively studied [19], where the tool's traverse speed is chosen so that local (i.e. MSF) errors are attenuated, but the tool adapts to the global form.

Returning to rigid tools, there is a specific 'window' where they can be successfully applied to aspheric or freeform surface. This corresponds to the case where the misfit between the tool and part, over the tool-path, is not larger than the size of the selected loose-abrasive grits [20].

In this paper, we report a novel method of applying aspheric rigid smoothing tool to aspheric surfaces to remove MSF errors. The work was organised, as shown in Fig. 1, into three packages. The first row of Fig. 1 showed work flow of experiment parameters optimisation. The second row showed the work flow of generating an aspheric surface to be processed. The third row showed work flow of tool parameter optimisation and fabrication.

\section{Background}

It is recognised [13] that several factors will lead to MSF errors, including misfit, toolpath track spacing, tool pressure, errors in tool-orientation, etc. The purpose of the simulation reported here was to predict MSF errors that arise from such factors and to optimise experimental parameters to minimise MSF content. The target is, through optimisation, to reduce the misfit to a level comparable to the polishing grit sizes.
It is widely accepted that a tool's Influence Function (IF) is a good representation of its removal characteristic. It combines effects of pressure distribution, relative tool speed, local dwell time and chemical reactions that occurred. For a standard compliant polishing tool, the IF can be considered constant, whilst the local removal is proportional to the local dwell time. For a rigid tool, especially when it is processing an aspheric surface, the IFs will vary since the misfit between the tool and the surface changes constantly. This variation of misfit leads to dynamic pressure distribution thus change not only the shape but also the volume of the IFs.

To obtain the contact condition, COMSOL Multiphysics, a finite element analysis software package, was used in this work. The solid mechanics module, under the structural mechanics branch in COMSOL Multiphysics, was intended for general solid structural mechanics analysis. In the solid mechanics module, the contact pair can be added and the contact analysis interface can be conducted. In addition, linear material mode, non-linear material model or user-defined material model can be introduced into the simulation process.

In this work, polishing pitch No. 73 from Satisloh was used for the active surface of the tool pad. According to the special physical properties of pitch, and complex surface contact conditions in polishing, some assumptions were made to simply the simulation process and to reduce calculation time. These, based on our previous experimental knowledge, were as follows:- (i) It was assumed that the pitch under our experimental conditions was an elastic material, which meant that the linear material model could be used in the simulation. The polishing pitch was in reality viscoelastic, but could be considered as elastic when the temperature was below $40{ }^{\circ} \mathrm{C}$. In our polishing process, the tool rotation speed was $60 \mathrm{rpm}$ and there was adequate slurry to cool the pitch tool. (ii)

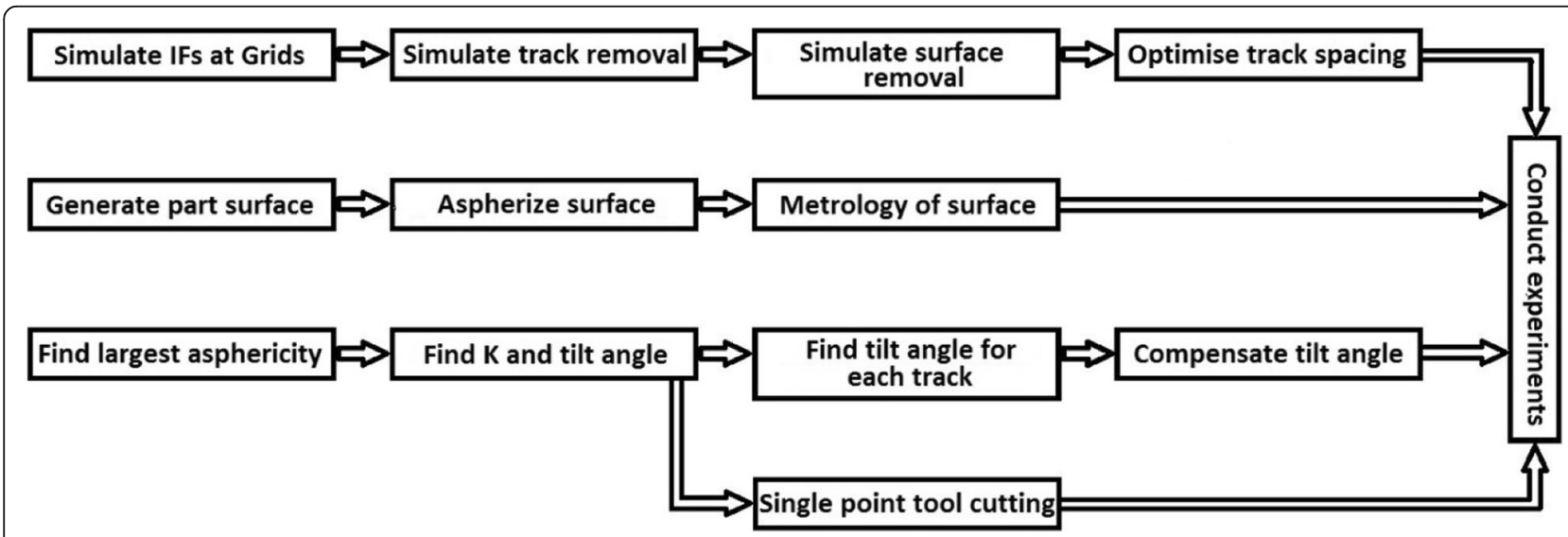

Fig. 1 Work flowchart of simulation, part preparation and tool cutting 
It was assumed that the polishing force was uniformly applied to the pitch tool. The metal holder for the pitch was cemented to the surface of a standard Precessions $^{\mathrm{Tw}}$ polishing-bonnet, which was pressurized as usual. The bonnet then provided a spring function, so that the force on the pitch holder was uniformly distribution. (3) It was assumed that the pitch holder was a rigid body in the model. Under the modest loading conditions for polishing, any deformation could be neglected.

\section{Model description}

Figure 2a shows a schematic diagram of the pitch tool contacting with the part, which was built according to our experimental setup. Given that there were different misfit conditions, no symmetry was applied in this mode. Both the aspheric part and aspheric tool were built up according to the misfit calculation software. Linear elastic material modelling was used, and the specific pair of contact interface was added. According to the technology datasheet, the material properties of pitch tool are assigned as follows: Young's Modulus E = $2 \mathrm{GPa}$, Poisson's ratio $\mathrm{v}=0.4$, and density $p=1.8 \mathrm{~g} / \mathrm{cm} 3$. The substrate was silica glass and the material properties were the following: Young's Modulus E $=77 \mathrm{GPa}$, Poisson's ratio $\mathrm{v}=0.17$, and density $p=2.2 \mathrm{~g} / \mathrm{cm} 3$.

Based on the elastic mechanics theory, the contact process could be express as the following governing equations

$$
\begin{aligned}
& \nabla \cdot(F \cdot S)^{T}+\boldsymbol{F}_{\boldsymbol{v}} \\
& F=\boldsymbol{I}+\nabla \boldsymbol{u}
\end{aligned}
$$

Where $\mathrm{F}$ denoted the deformation gradient, $\mathrm{F}_{\mathrm{v}}$ denoted the volume force, $\mathrm{u}$ was the displacement, $\nabla$ represented the differential operator, $\mathrm{S}$ denoted the Second Piola-
Kirchhoff stress, I denoted the unit matrix and $u$ was the displacement.

To solve the governing equations, the boundary conditions must be introduced into the model, which should be consistent with the experiments. The load was assigned to the upper surface of the pitch holder as a uniformly distributed force, which was $8 \mathrm{~N}$ according to our previous studies. The bottom surface of the substrate was fixed, where there was no displacement. The contact surfaces included the upper surface of the part and the lower surface of the pitch tool, which was described using the Augmented Lagrangian Method. In the contact pair, the surface of pitch was assigned as destination surface and the surface of the part was assigned as the source surface.

\section{Mesh and solver settings}

Due to geometry changes for different misfits, the mesh settings were optimised for each simulation. Overall, the Free Tetrahedral mesh was applied and refined at contact surfaces. Based on our experience, the maximum element size was less than $1 \mathrm{~mm}$ and the maximum element growth rate was less than 1.1 in the contact zone. A representative meshing result was shown in Fig. 2b. Stationary solver was used in this model. To improve the calculation efficiency, segregated solution approach was chosen to split solving process into two steps.

To simulate contact pressure, the amount of misfit between the tool and the part had to be calculated. This can be seen in Fig. 4. The misfit was derived from the difference of corresponding area between the tool and the part.

The surface of pitch tool and optic part in contacting area was expression by conic surface formula below.

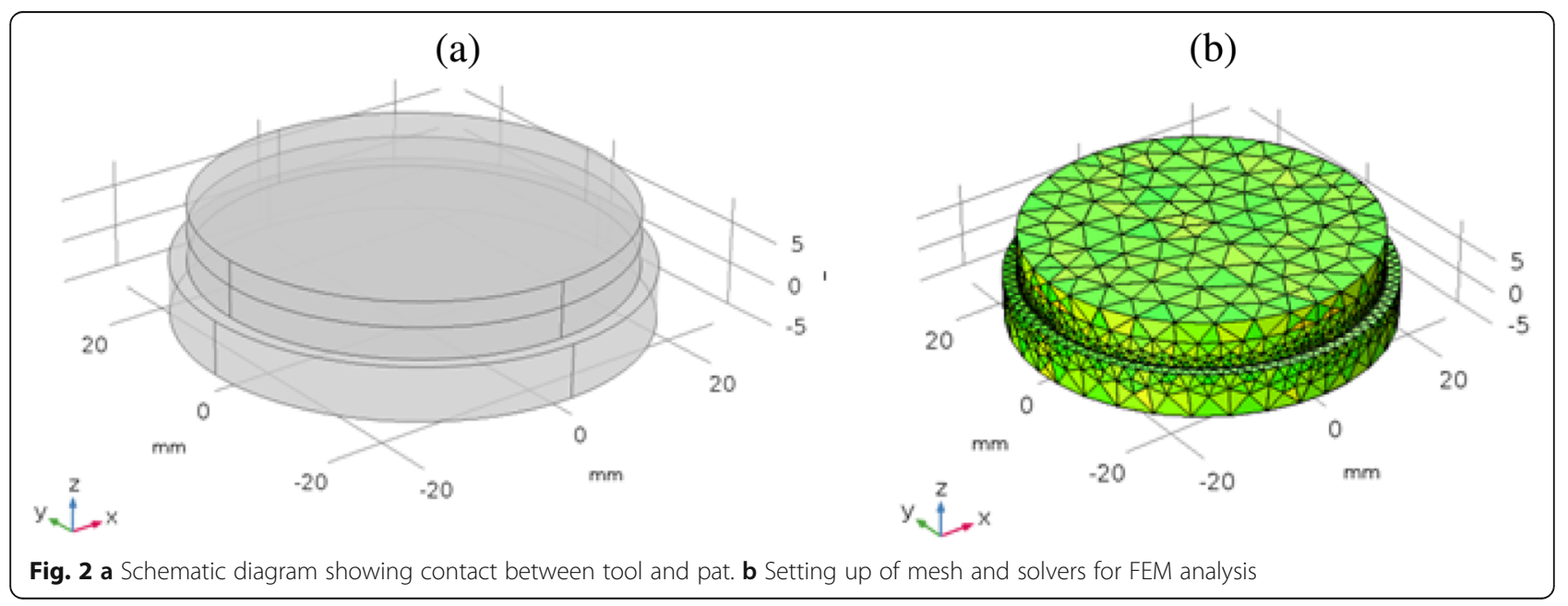




$$
z=\frac{t}{b+\sqrt{b^{2}-a t}}
$$

In the formula, $z$ was the surface distance in Cartesian coordinates. Other parameters were described as follows:

$$
\begin{aligned}
& a=c\left(1+k \cos ^{2} \theta\right) \\
& b=\frac{1}{\sqrt{1+k \sin ^{2} \theta}}-c k \sin \theta \cos \theta x \\
& t=c\left(1+k \sin ^{2} \theta\right) x^{2}+c y^{2}
\end{aligned}
$$

Where $x$ and $y$ were the coordinate values in the conic surface. $c$ and $k$ were the curvature of the vertex the conic constant respectively.

With the amount of misfit calculated as shown in Fig. 3, the pressure distribution between the tool and the part were plotted at different radial locations, as shown in Fig. 4. It can be seen that, with the misfit error increasing with radial distance, the pressure distribution changed considerably. This would lead to dynamically-changing IFs as the tool moved along the surface (Fig. 5).

\section{Optimisation of experimental parameters}

The aim was to design a process to eliminate the MSF errors from previous process-steps, but to leave a minimum signature of its own. The control of MSFs was through the optimisation of parameters such as track spacing and tool pressure. This simulation of experimental parameters on the processed surface was through the application of IFs at their locations. The benefit of this simulation was that much less experimental efforts and time be saved. Figure 6 showed the flowchart of the simulation procedure. The distribution of control points were firstly made considering the user's input, such as the part's size, track and point spacing, etc. These points were the calculation points where the IFs are simulated (left column of Fig. 6). The material removal was the convolution of the tool's local dwell time and influence function. The IFs were dynamic both in shape and volume so that all the local IFs needed to be simulated to predict the surface removal (mid column of Fig. 6). These would lead to intensive workload both for simulation of pressure distribution and material removal. To simply this, the trend of the variation of the IFs had been studied and interpolation had been introduced to predict some of the IFs. After the simulation of material removal of the whole part's surface, transacts of the surface maps would be obtained to analyses MSF error terms.

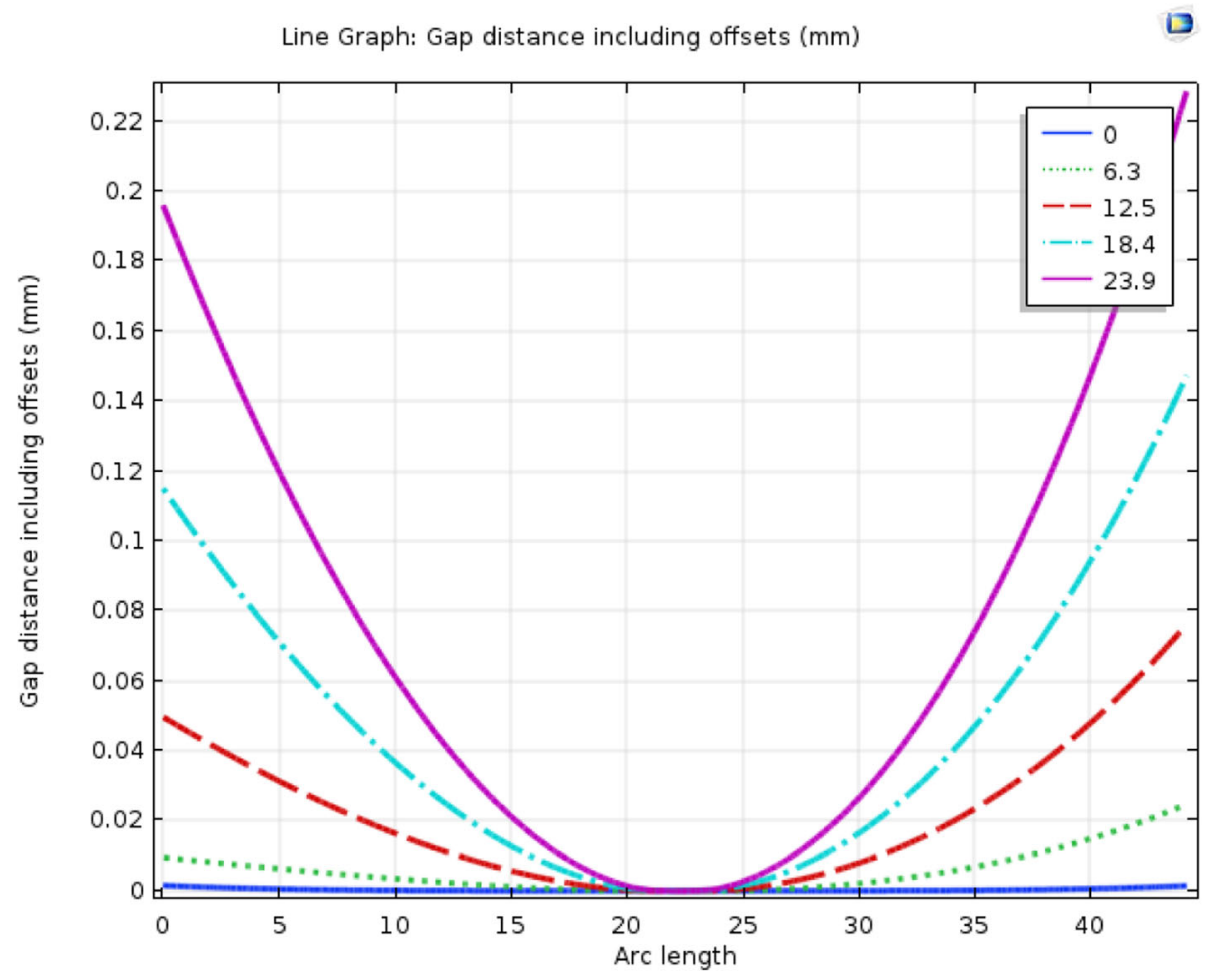

Fig. 3 Amount of misfit (PV) between the tool and the part of different radial distance 


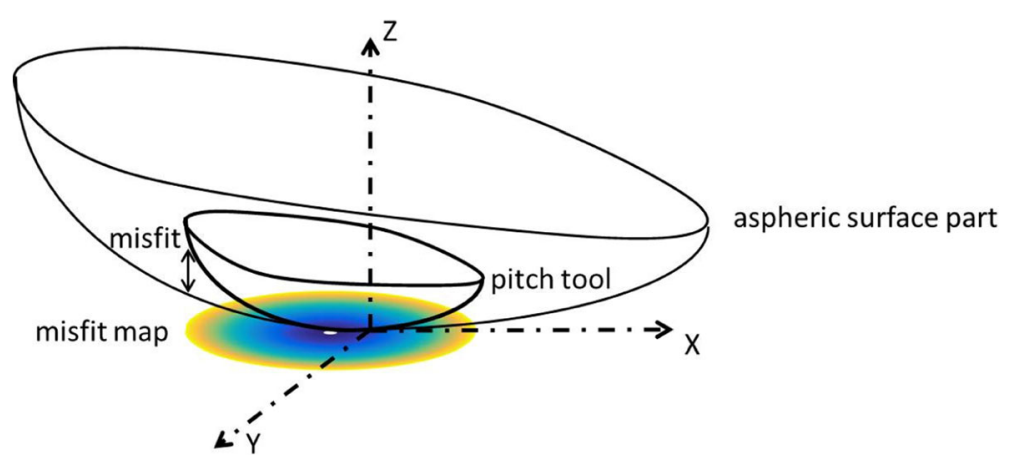

Fig. 4 Schematic diagram showing misfit between the tool and the part

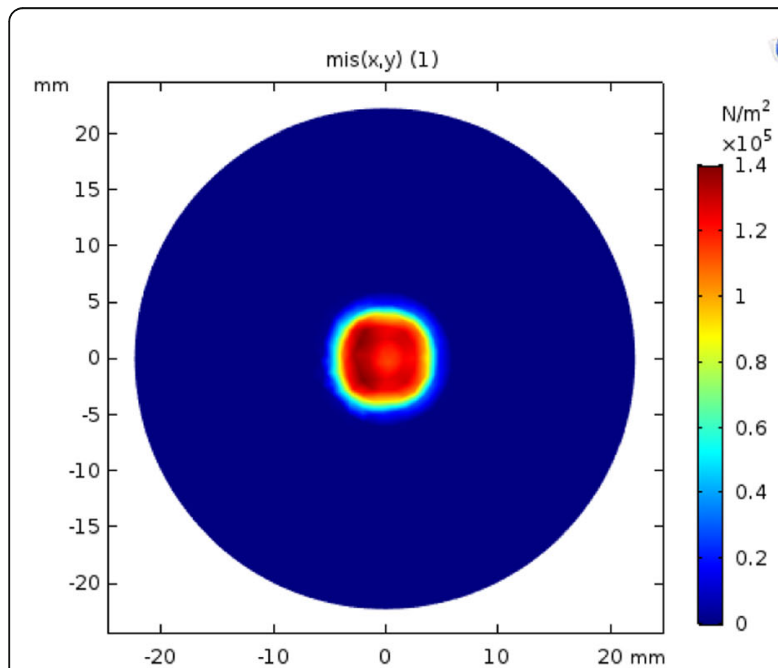

(a) Radial distance $0 \mathrm{~mm}$

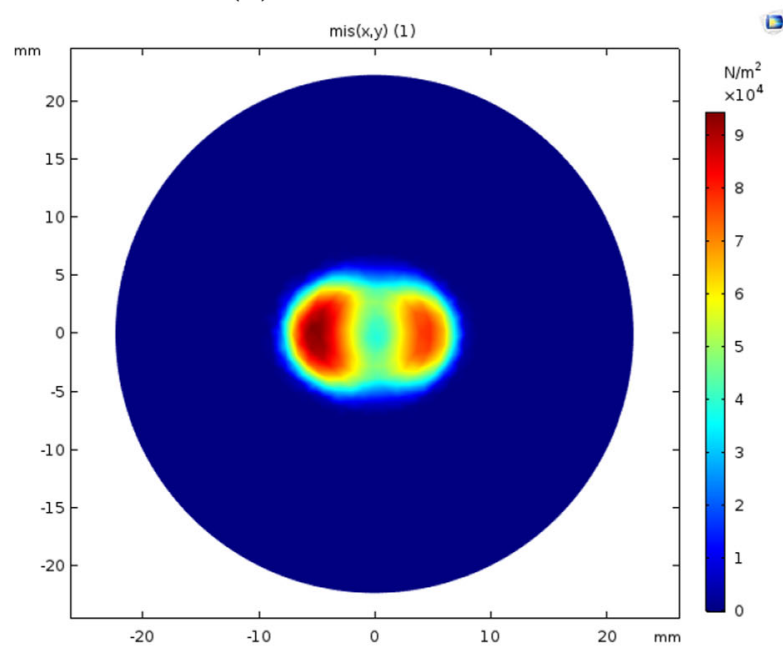

(c) Radial distance $50 \mathrm{~mm}$

0

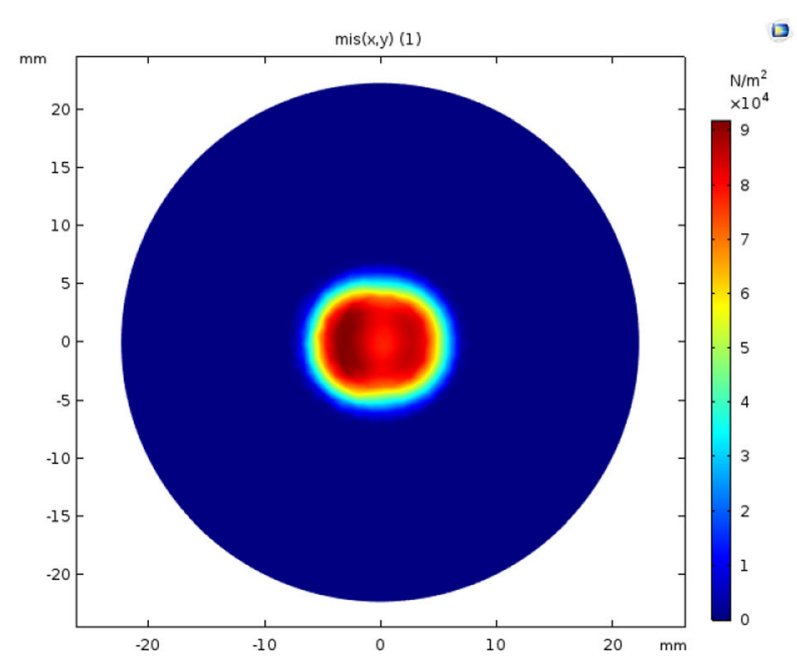

(b) Radial distance $30 \mathrm{~mm}$

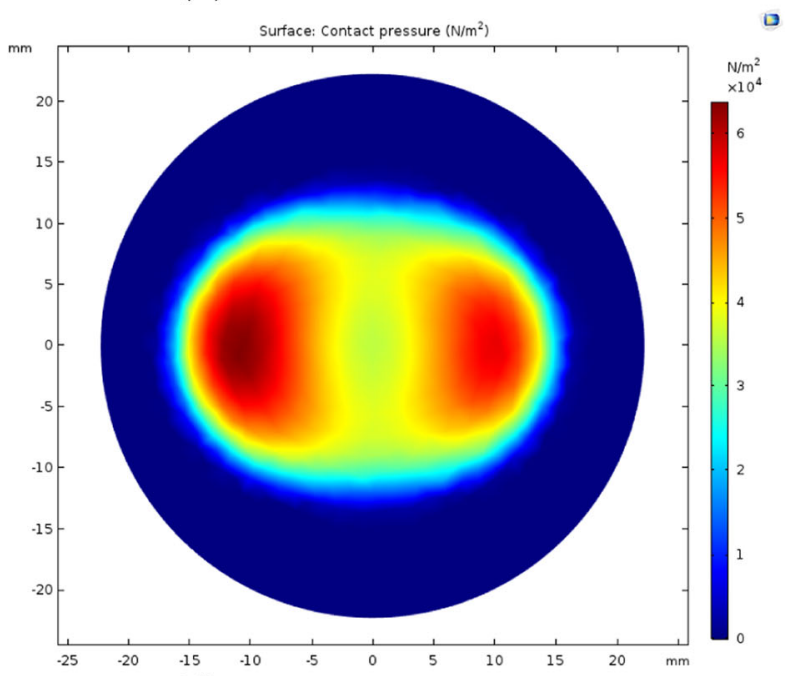

(d) Radial distance $100 \mathrm{~mm}$

Fig. 5 FEM simulation of pressure distribution between the tool and the part at varied locations. a Radial distance $0 \mathrm{~mm}$. b Radial distance 30 mm. c Radial distance $50 \mathrm{~mm}$. d Radial distance $100 \mathrm{~mm}$ 


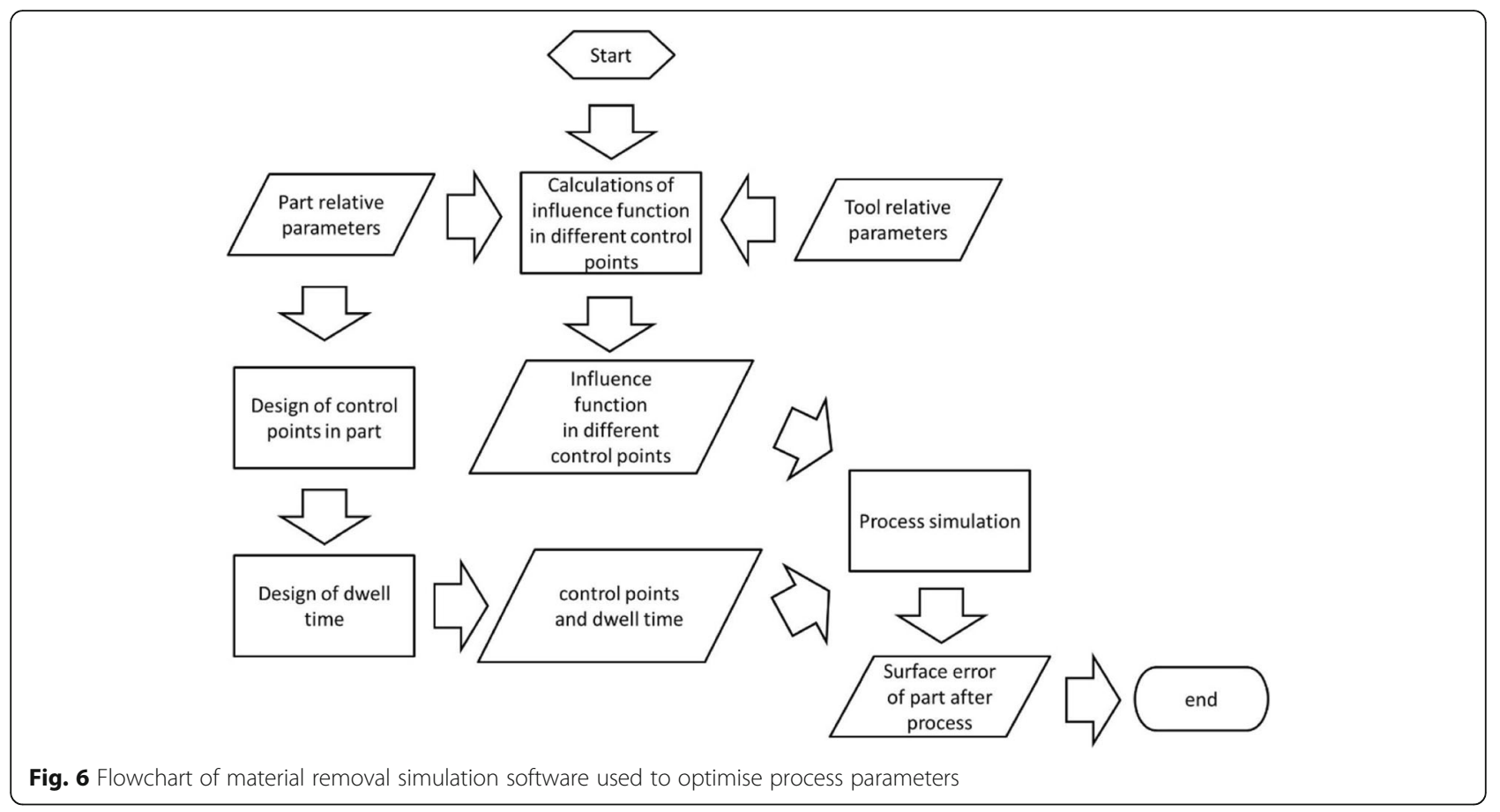

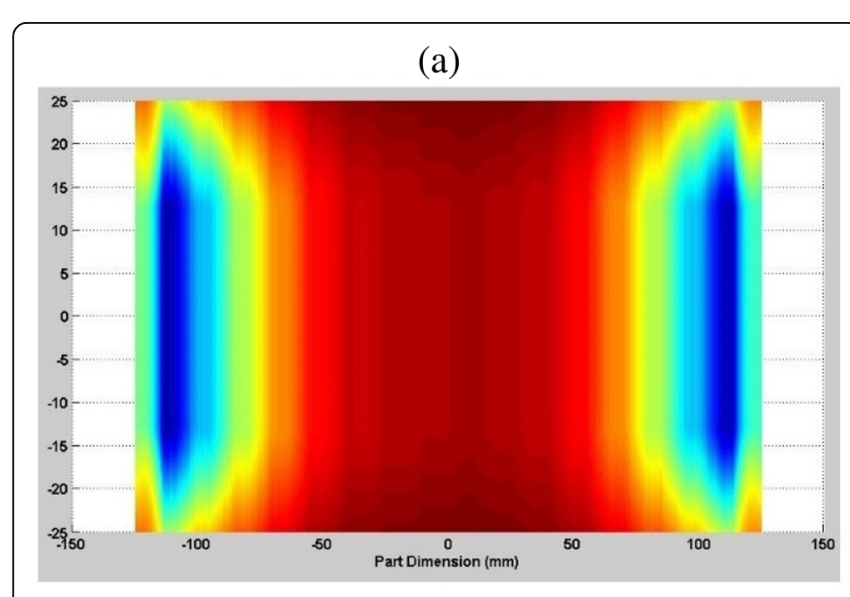

(c)

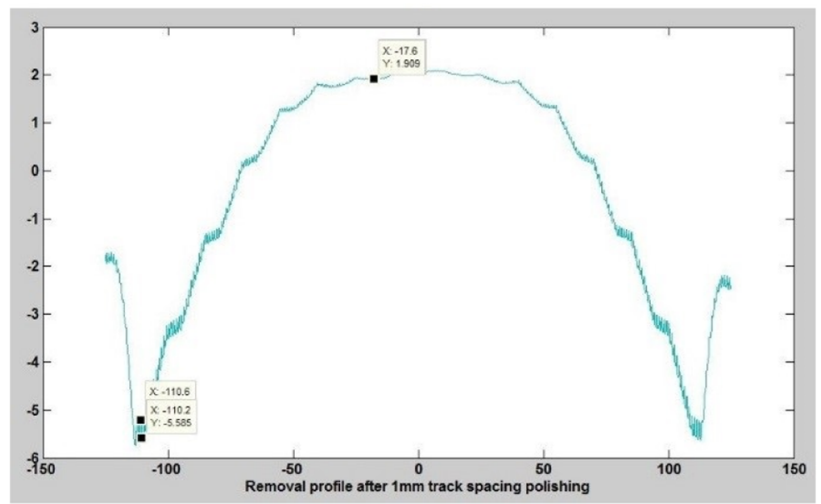

(b)

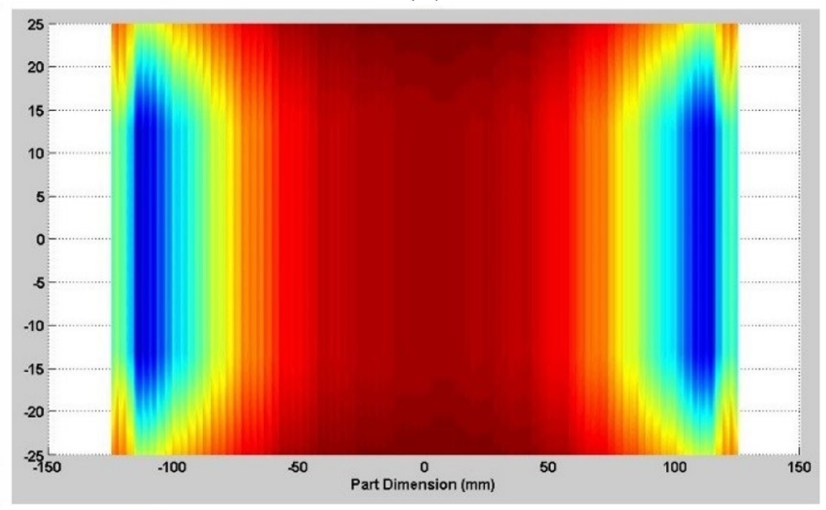

(d)

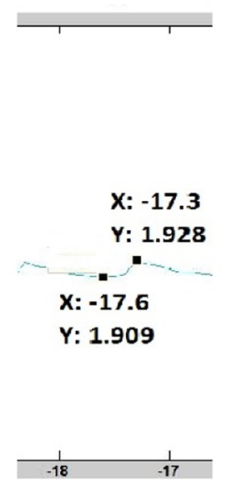

(e)

(f)

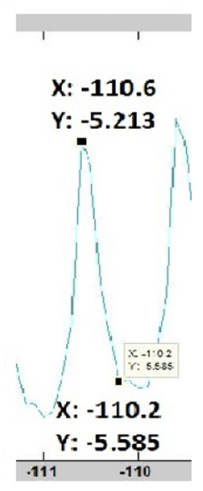

Fig. 7 Simulation results of material removal with: a track spacing of $1 \mathrm{~mm}$, $\mathbf{b}$ tracking spacing of $3 \mathrm{~mm}$, $\mathbf{c}$ profile with track spacing of $1 \mathrm{~mm}$, $\mathbf{d}$ MSF at central region, e MSF at edge region, $\mathbf{f}$ MSF of track spacing of $3 \mathrm{~mm}$ at edge region 


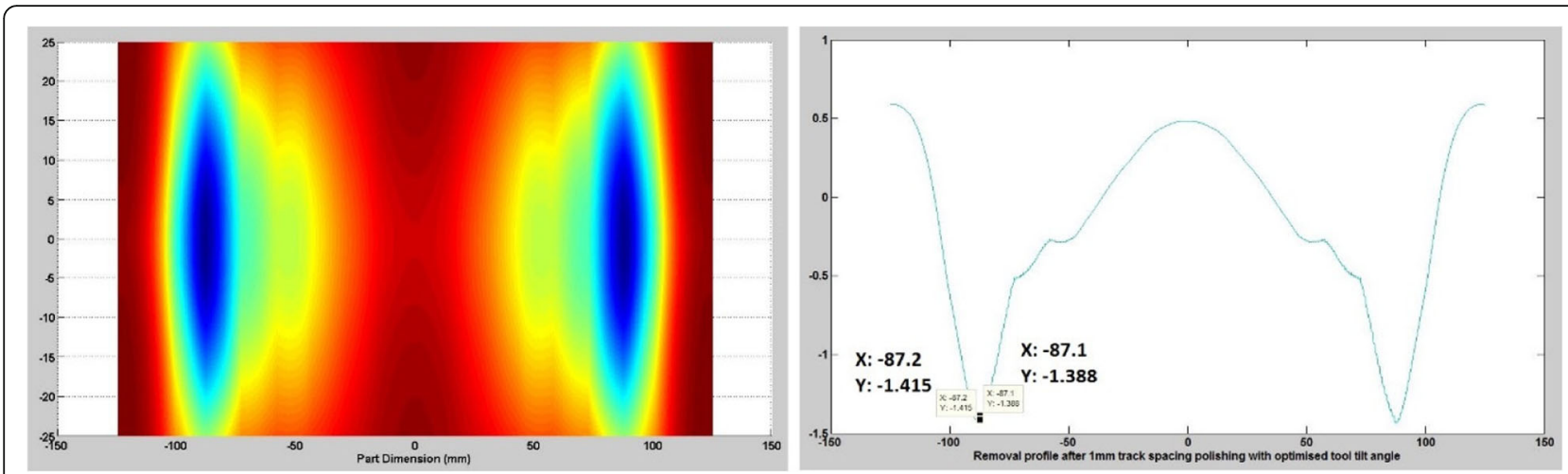

Fig. 8 Simulation results of material removal with $1 \mathrm{~mm}$ track spacing and optimised tool tilt angle

In Fig. 7 showed simulation results when using a spherical smoothing tool of base radius $270 \mathrm{~mm}$. In this simulation, the part's surface data was generated to be aspheric having a base radius of $270 \mathrm{~mm}$ and a departure of $100 \mu \mathrm{m}$ at the edges when it was osculating the ROC $=270 \mathrm{~mm}$ sphere at the centre. The simulation region was chosen to be 260 by $50 \mathrm{~mm}$ as representative of the real part but to save calculation time in reduced width. Firstly, it was shown that toolpath's track spacing was an important factor of controlling MSFs. (a) and (b) were the polishing removal maps when track spacing were of $1 \mathrm{~mm}$ and $3 \mathrm{~mm}$. It could be seen that the MSF errors, when using $1 \mathrm{~mm}$ track spacing were $19 \mathrm{~nm}$ at central region and $372 \mathrm{~nm}$ at edge region. With $3 \mathrm{~mm}$ track spacing, the MSF errors would be $49 \mathrm{~nm}$ at central region and $404 \mathrm{~nm}$ at edge region. These values reflected that both toolpath's track spacing and tool's misfit were going to affect the MSF errors. At central region, when tool's misfit were small, the MSF had relatively smaller PV values when other than those at edge regions where there were bigger misfits due to large asphericity. When the track spacing grew larger, the MSF errors became bigger even at the same region. All these provides guidance of experimental parameters when a MSF target is specified (Fig. 8).

Further to use a spherical tool, a simulation of using an aspheric tool was conducted with the tool's conic coefficient and tilt angle had been optimised. The aim of using an aspheric smoothing tool is to minimise the misfit between the tool and the part, thus reduce the MSF errors that were caused by the misfit. This work was conducted into two steps: calculation of tool's conic constant and optimisation of tool's tilt angle. The first step is to find an aspheric shape of the tool through a suitable choice of conic constant that will enable the tool to have a shape that will cover the local shape to the aspheric part. The second step is to choose a tilt angle of the tool when it moves onto the part's surface that will ensure the misfit is minimum. It was shown that by using an aspheric tool, the PV of MSF errors can be reduced from $372 \mathrm{~nm}$ from using a spherical tool to 27 $\mathrm{nm}$ by using the optimised aspheric tool.

\section{Results and discussion \\ Part and tool preparation}

To verify the effectiveness of the aspheric smoothing tool and of the simulation result, the part was generated and polished to be the same prescription of the part in the simulation. This was achieved by polishing it firstly into a spherical surface of ROC $270 \mathrm{~mm}$. To turn this surface into an asphere, a dummy error map was used for corrective polishing. The part was measured using Hexagon Absolute Arm (Type: RA-7512-4) after each polishing run. The data was fitted by least square method and the conic constant found out to be 0.1725 with base radius of $270 \mathrm{~mm}$ after 5th polishing run. To prepare the tool, the best conic constant of the pitch tool was optimised from minimising misfit between the tool and the part, is as shown in Table 1 below. It defines best conic constant which has minimum RMS of misfit in set range of tool's radial distance (about from $40 \mathrm{~mm}$ to $40 \mathrm{~mm}$ ).

Table 1 Best conic constant of pitch tool at different radial distance of the part

\begin{tabular}{lllllll}
\hline Part Radial Distance $(\mathrm{mm})$ & 0 & 25 & 50 & 75 & 100 & 125 \\
Tool Best Conic Constant $(\mathrm{mm})$ & 0.18182 & 0.060606 & 0.18182 & 0.66667 & 1.5152 & 2.6061 \\
Max RMS $(\mu \mathrm{m})$ & 10.7752 & 2.5171 & 1.9912 & 2.2716 & 11.6378 & 21.8903 \\
\hline
\end{tabular}




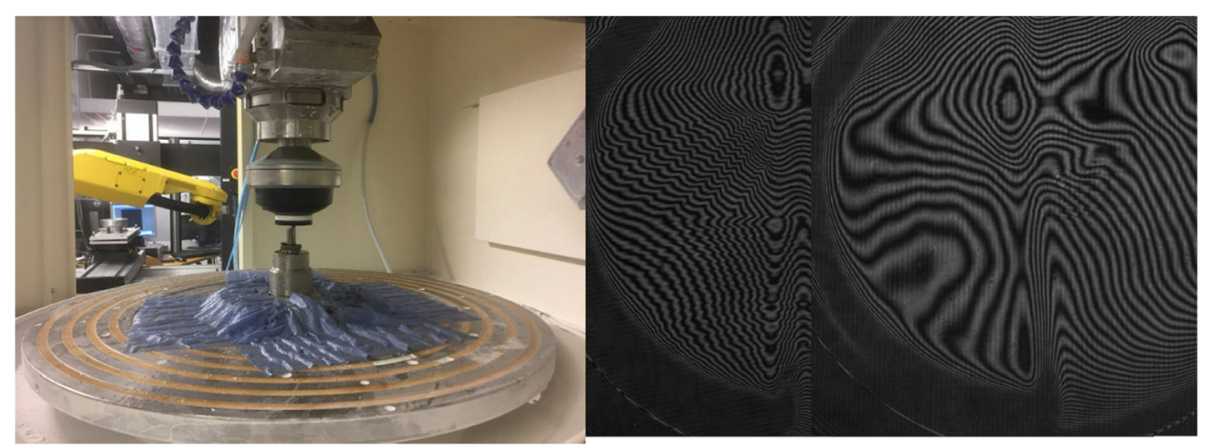

Fig. 9 (left) Pitch contact surface machined to designed aspheric surface with single point cutter. (middle) A section of the part's surface with MSFs imprinted by spherical smoothing tool. (right) A section of the surface where MSFs has been removed by using aspheric smoothing tool with optimised tilt angle

\section{Polishing experiments and results}

In the first experiment, the tool was used at pole down position at all places so that the tilt angle was 0 . The metrology of the MSF errors can only be seen on an interferometer. But the error phase map cannot be obtained since the departure of the surface from a sphere was beyond the capability of the interferometer. Even so, the MSF errors can still be observed through fringe patterns. Although accurate calculation of the amplitude of the MSF errors can be done by comparing the 'spikes' with the fringes' spacing. We have taken screen shots of the fringe pattern just to demonstrate the existence of them. This was adequate to verify the efficiency of the effectiveness of the aspheric smoothing tool.

\section{Discussion}

The fringes of Fig. 9 (middle) which shows signatures of MSFs. This is due to the large misfit of the spherical tool with the local aspheric surface. The level of the MSFs generated are calculated to be $388 \mathrm{~nm}$ at edge zone, which was close to the simulation result of $372 \mathrm{~nm}$. We have also noticed that the levels of MSF errors were proportional to the levels of misfits between the tool and the aspheric part surface, which also agreed to the simulation. The aspheric tool was then used to smooth the part's surface. Differ from the spherical tool, the aspheric tool's tilt angle had been compensated manually. The tilt angles calculated to be able to produce the minimum misfit at each surface point had been added to machine file. From the experimental result, shown in Fig. 9 (right), we can noticed that those MSFs had been removed by using this aspheric smooth tool.

\section{Conclusion}

We have experimentally demonstrated that an aspheric rigid smoothing tool can be applied to remove MSEs on aspheric surfaces. Software has been developed to calculate compensation tilt angle and to optimise experimental results. The MSF errors removed are generated from polishing process. The same principle can be applied on MSF errors in other process with bespoke tooling and different abrasives. For example, MSF errors from cup grinding can be treated with brass button tool and with larger-grit Aluminum Oxide slurry. The shape of the tool and the application tilt angles have to be optimised as described in this paper so that best-fit contact can be achieved.

\section{Acknowledgements \\ Not applicable.}

\section{Authors' contributions}

GY supervised the whole work and technical assistant on tool making and part polishing. LW optimised the experimental parameters and tool's tilt angle and conic constant. XS used FEM method to simulate influence functions. YL conducted tool generation and surface metrology. KW conducted experiments of producing aspheric part and removal of MSF errors. HL provided consultancy on simulation of IFs and selection of materials for tool making. DW provided consultancy on experimental methods regarding tool and part mismatch. All authors read and approved the final manuscript.

\section{Authors' information}

GY has a PhD in Optics and is a member of Optical Society of America. He is a senior research fellow at the University of Huddersfield and has many years of experience in process larger aperture aspheric optical surfaces.

\section{Funding}

This research is supported by the University of Huddersfield and the Chinese Scholarship Council.

Availability of data and materials Not applicable.

\section{Competing interests}

The authors declare that they have no competing interests.

\section{Author details}

${ }^{1}$ National Facility for Ultra Precision Surfaces, OpTIC Centre, University of Huddersfield, St. Asaph Business Park, Ffordd William Morgan, St Asaph, North Wales LL17 0JD, UK. ${ }^{2}$ Shanghai Institute of Optics and Fine Mechanics, 
The Chinese Academic of Science, Shanghai, China. ${ }^{3}$ Center for Precision Engineering, Harbin Institute of Technology, Harbin, China. ${ }^{4}$ Key Laboratory of Optical System Advanced Manufacturing Technology, Changchun Institute of Optics, Fine Mechanics and Physics, The Chinese Academic of Science, Changchun, China. ${ }^{5}$ Research Center for Space Optical Engineering, Harbin Institute of Technology, Harbin, China. ${ }^{6}$ Department of Physics and Astronomy, University College, Gower St, London WC1E 6BT, UK. 'ZZeeko Ltd, 4 Vulcan Court, Vulcan Way, Coalville, Leicestershire LE67 3FW, UK.

Received: 24 January 2019 Accepted: 23 July 2019

Published online: 02 August 2019

\section{References}

1. Huang, H., Gong, Z.M., Chen, X.Q., Zhou, L.: Robotic grinding and polishing for turbine-vane overhaul. J. Mater. Process. Tech. 127(2), 140-145 (2002)

2. Walker, D.D., Beaucamp, A.T.H., Doubrovski, V., Dunn, C., Evans, R., Freeman, R., McCavana, G., Morton, R., Riley, D., Simms, J., Yu, G., Wei, X: Commissioning of the first precessions $1.2 \mathrm{~m}$ CNC polishing machines for large optics. In: Current developments in lens design and optical engineering VII, vol. 6288, (2006)

3. Walker, D., Yu, G.Y., Li, H.Y., Messelink, W., Evans, R., Beaucamp, A.: Edges in CNC polishing: from mirror-segments towards semiconductors, paper 1: edges on processing the global surface. Opt. Express. 20(18), 19787-19798 (2012)

4. Sugawara, J., Kamiya, T., Mikashima, B.: Polishing aspheric mirrors of zerothermal expansion cordierite ceramics (NEXCERA) for space telescope. In Material technologies and applications to optics, structures, components, and sub-systems III, vol. 10372, (2017)

5. Han, S.J., Choi, J.K., Lee, S.W., Choi, H.Z.: A study of an ultra-precision CNC polishing system. Key Eng. Mat. 257-258, 395-400 (2004)

6. Martin, H.M., Allen, R.G., Burge, J.H., Davis, J.M., Davison, W.B., Johns, M., Kim, D.W., Kingsley, J.S., Law, K., Lutz, R.D., Strittmatter, P.A., Su, P., Tuell, M.T. West, S.C., Zhou, P.: Production of primary mirror segments for the giant Magellan telescope. In: Advances in optical and mechanical technologies for telescopes and instrumentation, vol. 9151, (2014)

7. Oota, T., Negishi, M., Shinonaga, H., Gomi, A., Tanaka, Y., Akutsu, K., Otsuka, I., Mochizuki, S., Iye, M., Yamashita, T.: High volume production trial of Mirror segments for the thirty meter telescope. In: Ground-based and airborne telescopes V, vol. 9145, (2014)

8. Tamkin, J.M., Milster, T.D.: Effects of structured mid-spatial frequency surface errors on image performance. Appl. Opt. 49(33), 6522-6536 (2010)

9. Achilles, K., Uhlendorf, K., Ochse, D.: Tolerancing the impact of midspatial frequency surface errors of lenses on distortion and image homogeneity. In: Optical systems design 2015: optical design and engineering $\mathrm{VI}$, vol. 9626, (2015)

10. Maksimovic, M:: Optical tolerancing of structured mid-spatial frequency errors on free-form surfaces using anisotropic radial basis functions. In: Optical systems design 2015: optical design and engineering VI, vol. 9626, (2015)

11. Del Hoyo, J., Choi, H., Burge, J.H., Kim, G.H., Kim, D.W.: Experimental power spectral density analysis for mid- to high-spatial frequency surface error control. Appl. Opt. 56(18), 5258-5267 (2017)

12. Dai, Y.F., Shi, F., Peng, X.Q., Li, S.Y.: Restraint of mid-spatial frequency error in magneto-rheological finishing (MRF) process by maximum entropy method. Sci. China Ser. E. 52(10), 3092-3097 (2009)

13. $\mathrm{Yu}, \mathrm{G}$., Li, H., Walker, D.: Removal of mid spatial-frequency features in mirror segments. J. Eur. Opt. Soc.-Rapid. 6, 11044-4 (2011)

14. Nie, X.Q., Li, S.Y., Hu, H., Li, Q.: Control of mid-spatial frequency errors considering the pad groove feature in smoothing polishing process. Appl. Opt. 53(28), 6332-6339 (2014)

15. Wang, T., Cheng, H.B., Yang, H., Wu, W.T., Tam, H.Y.: Controlling mid-spatial frequency errors in magnetorheological jet polishing with a simple vertical model. Appl. Opt. 54(21), 6433-6440 (2015)

16. Kim, D.W., Burge, J.H.: Rigid conformal polishing tool using non-linear viscoelastic effect. Opt. Express. 18(3), 2242-2257 (2010)

17. Tuell, M.T., Burge, J.H., Anderson, B.: Aspheric optics: smoothing the ripples with semiflexible tools. Opt. Eng. 41(7), 1473-1474 (2002)

18. Anderson, D.S., Angel, J.R.P., Burge, J.H., Davison, W.B., Derigne, S.T., Hille, B.B., Ketelsen, D.A., Kittrell, W.C., Martin, H.M., Nagel, R.H., Trebisky, T.J., West, S.C., Young, R.S.: Stressed-lap polishing of 3.5-M F 1.5 and 1.
8-M F 1.0 mirrors. In: Advanced optical manufacturing and testing II, vol. 1531, pp. 260-269 (1992)

19. Kim, D.W., Park, W.H., Kim, S.W., Burge, J.H.: Parametric modeling of edge effects for polishing tool influence functions. Opt. Express. 17(7), 5656-5665 (2009)

20. Walker, D., Hsing-Yu, W., Yu, G.Y., Li, H.Y., Zhang, W., Lu, C.L.: Insight into aspheric misfit with hard tools: mapping the island of low mid-spatial frequencies. Appl. Opt. 56(36), 9925-9931 (2017)

\section{Publisher's Note}

Springer Nature remains neutral with regard to jurisdictional claims in published maps and institutional affiliations.

\section{Submit your manuscript to a SpringerOpen ${ }^{\circ}$ journal and benefit from:}

- Convenient online submission

- Rigorous peer review

- Open access: articles freely available online

- High visibility within the field

- Retaining the copyright to your article

Submit your next manuscript at $\boldsymbol{\nabla}$ springeropen.com 